number of patients with HCAIs. The most frequent infections were clinically defined pneumonia (30.9\%) and bloodstream infections (20.1\%). The most frequently isolated microorganism was Pseudomonas aeruginosa. Conclusions: The prevalence of HCAI was $12.2 \%$. The most frequent HCAIs were pneumonia and bloodstream infection.

Funding: No

Disclosures: None

Antimicrobial Stewardship \& Healthcare Epidemiology 2021;1(Suppl. S1):s78-s79

doi:10.1017/ash.2021.154

\section{Presentation Type:}

Poster Presentation

Subject Category: Surveillance/Public Health

Multifacility Outbreak of NDM/OXA-23-Producing Acinetobacter baumannii in California, 2020-2021

Diana Holden; Tisha Mitsunaga; Denise Sanford; Tanya Fryer; June Nash; Emily Schneider; Rituparna Mukhopadhyay; Erin Epson and Matthew Sylvester

Background: NDM/OXA-23 carbapenemase-producing Acinetobacter baumannii isolates have been reported worldwide, but rarely in the United States. A California acute-care hospital (ACH) A identified 3 patients with pan-nonsusceptible A. baumannii during May-June 2020, prompting a public health investigation to prevent further transmission among the regional healthcare network. Methods: A clinical isolate was defined as NDM/OXA-23-producing A. baumannii from a patient at $\mathrm{ACH} \mathrm{A}$ or B, or an epidemiologically linked patient identified through colonization screening during May 2020-January 2021. ACHs A and B are sentinel sites for carbapenem-resistant $A$. baumannii surveillance through the Antibiotic Resistance Laboratory Network (AR Lab Network), where isolates are tested for carbapenemase genes. The California Department of Public Health with 3 local health departments conducted an epidemiological investigation, contact tracing, colonization screening, and wholegenome sequencing (WGS). Results: In total, 11 cases were identified during May 2020-January 2021, including 3 cases at ACH A during May-June 2020, and 8 additional cases during November 2020-January 2021: 5 at $\mathrm{ACH} A, 1$ at $\mathrm{ACH}$ B, and 2 at skilled nursing facility (SNF) A. Isolates from ACHs A and B were identified through testing at the AR Lab Network. Of the 11 patients (including the index patient), 4 had exposure at SNF A, where 2 cases were identified through colonization screening. Screening conducted at ACH A and 5 other long-term care facilities (LTCFs) identified no additional cases. WGS results for the first 8 cases identified showed 2-13 single-nucleotide polymorphism differences. Antibiotic resistance genes for all isolates sequenced included NDM-1 and OXA-23. On-site assessments related to a COVID-19 outbreak conducted at ACH A identified infection control gaps. Conclusions: Hospital participation in public health laboratory surveillance allows early detection of novel multidrug-resistant organisms (MDROs), which enabled outbreak identification and public health response. A high COVID-19 burden and related changes in infection control practices have been associated with MDRO transmission elsewhere in California. This factor might have contributed to spread at ACH A and hampered earlier screening efforts at SNF A, likely leading to undetected transmission. Extensive movement of positive patients among a regional healthcare network including at least $6 \mathrm{ACHs}$ and 7 LTCFs likely contributed to the prolonged duration of this outbreak. This investigation highlights the importance of enhanced novel MDRO surveillance strategies coupled with strong infection prevention and control practices as important factors in identifying outbreaks and preventing further transmission in regional networks.

Funding: No

Disclosures: None

Antimicrobial Stewardship \& Healthcare Epidemiology 2021;1(Suppl. S1):s79

doi:10.1017/ash.2021.155
Presentation Type:

Poster Presentation

Subject Category: Surveillance/Public Health

Surveillance of Candidemia in Connecticut: An Epidemiological Comparison Between Two Periods

Johanna Gleason-Vergados; David Banach; Paula Clogher and James Meek

Background: Candidemia is the fourth most common bloodstream infection in hospitalized patients in the United States, with an attributable mortality rate between $30 \%$ and $50 \%$. Understanding the epidemiology of candidemia is critical due to its prevalence and association with extended hospital stays, high treatment cost, and significant morbidity and mortality. In 2019 the Connecticut Department of Public Health deemed candidemia a mandatory reportable condition and began state-wide surveillance in conjunction with the CDC's Emerging Infections Program (EIP). Previously, the EIP had conducted population-based statewide surveillance of candidemia in Connecticut from 1998 to 2000, allowing an opportunity to assess how the epidemiology of candidemia has evolved. The goal of this study is to compare state-wide Connecticut EIP candidemia data from 2 periods (1998-2000 and 2019) to identify trends in infections and incidence, providing insight for potential improvements to current prevention measures and treatments. Methods: The sample population included all Connecticut residents aged $\geq 20$ who tested positive for a candidemia infection during 1998-2000 and 2019. Patients who had positive blood cultures for Candida spp but were $<20$ years old or were not Connecticut residents were excluded. Connecticut EIP candidemia case report forms from each time period were compared and matching fields were chosen as variables for univariate analysis to search for statistically significant differences. Selected variables include: Candida species present in blood culture, patient demographics, previous exposures to healthcare settings, length of stay, presence of central venous catheter (CVC), and location of the patient at diagnosis (community vs. hospital onset). De-identified patient-level information was provided by the EIP. Results: In total, 381 candidemia episodes from 1998-2000 were compared to 247 episodes in 2019. The proportion of C. albicans species in 1998-2000 was $49.9 \%$ and declined to $40.5 \%$ of cases in $2019(P=.02)$. Outcomes improved as well, with $65.2 \%$ of patients in 2019 having survived compared to $51.4 \%$ in $1998-$ $2000(P=.001)$. Other findings indicate that patients with candidemia in 2019 were less likely to have a central venous catheter, less likely to have undergone a recent surgery, and were more likely to have communityonset infection (all p < 0.05). Conclusions: The epidemiology of candidemia has changed over the past 20 years, with significant improvements in patient survival and a shift toward community-onset infections and non-

Table 1.

\begin{tabular}{|c|c|c|c|c|}
\hline & & \multicolumn{2}{|c|}{ YEAR } & \multirow[b]{2}{*}{ Chi-Square $P$-Value } \\
\hline & & $1999-2000$ & 2019 & \\
\hline \multirow{3}{*}{ Candida species present in blood culture } & C. albicans & $190(49.9 \%)$ & $100(40.5 \%)$ & \multirow{3}{*}{0.021} \\
\hline & \begin{tabular}{|l} 
Non-C. albicans \\
\end{tabular} & $191(50.1 \%)$ & $147(53.8 \%)$ & \\
\hline & Total & 381 & & \\
\hline \multirow{3}{*}{ Patient Outcome } & Died & $185(48.6 \%)$ & $86(34.8 \%)$ & \multirow{3}{*}{0.001} \\
\hline & Survived & $196(51.48)$ & $161(65.28)$ & \\
\hline & Total & & 247 & \\
\hline \multirow{3}{*}{ Length of Stay } & $<21$ days & $173(45.486)$ & $140(56.75)$ & \multirow{3}{*}{0.006} \\
\hline & 221 days & & & \\
\hline & $\frac{2.100 p_{3}}{\text { Total }}$ & $\frac{3013.060}{381}$ & $\frac{2475}{247}$ & \\
\hline \multirow{3}{*}{ Surgery 3 months preceding culture } & № & $176(46.25)$ & $180(72.966)$ & \multirow[b]{2}{*}{$<0.001$} \\
\hline & Yes & $205(53.8 \%)$ & $67(27.15)$ & \\
\hline & Total & $\begin{array}{c}381 \\
26(6.8 \%)\end{array}$ & $\frac{247}{118(47.85)}$ & \multirow{3}{*}{$<0.001$} \\
\hline \multirow{2}{*}{$\begin{array}{l}\text { Did the patient have a catheter at time of candidemia } \\
\text { episode }\end{array}$} & Yes & $355(93.286)$ & $129(52.28)$ & \\
\hline & Total & 381 & 247 & \\
\hline \multirow{3}{*}{ Hospital ward of patient at time of candidemia episode } & Inpatient & $367(96.3 \%)$ & $195(78.96)$ & \multirow{3}{*}{$<0.001$} \\
\hline & Outpatient & $14(3.796)$ & $52(21.1 \%)$ & \\
\hline & Total & 381 & 247 & \\
\hline \multirow{3}{*}{ Time from admission to culture } & $<17$ days & $255(66.9 \%)$ & $194(78.5 \%)$ & \multirow{3}{*}{0.002} \\
\hline & 217 days & $126(33.1 \%)$ & $53(21.5 \%)$ & \\
\hline & $\frac{T}{\text { Total }}$ & 381 & 247 & \\
\hline \multirow{3}{*}{ Number of comorbid conditions } & $<3$ conditions & $91(23.95)$ & $159(64.485)$ & \multirow{3}{*}{$<0.001$} \\
\hline & 23 conditions & $290(76.1 \%)$ & $88(35.68)$ & \\
\hline & Total & 381 & 247 & \\
\hline \multirow{2}{*}{ sex } & Female & 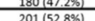 & & \multirow{2}{*}{0.118} \\
\hline & $\begin{array}{l}\text { Made } \\
\text { Total }\end{array}$ & $\frac{201528850}{381}$ & $\frac{146(59,10)}{247}$ & \\
\hline \multirow{5}{*}{ Age } & $20-44$ & $50(13.1 \%)$ & $38(15.485)$ & \multirow{2}{*}{0.425} \\
\hline & Other & $331(86.996)$ & $209(84.65)$ & \\
\hline & $45-64$ & $100(26.2 \%)$ & $80(32.4 \%)$ & \multirow{2}{*}{0.096} \\
\hline & Other & $281(73.8 \%)$ & $167(67.656)$ & \\
\hline & $65+$ & $\frac{231(60.6 \%)}{150.39848}$ & $129(52.25)$ & 0.038 \\
\hline
\end{tabular}

(c) The Author(s), 2021. Published by Cambridge University Press on behalf of The Society for Healthcare Epidemiology of America. This is an Open Access article, distributed under the terms of the Creative Commons Attribution licence (http://creativecommons.org/licenses/by/4.0/), which permits unrestricted re-use, distribution, and reproduction in any medium, provided the original work is properly cited. 